\title{
A PCR-based platform for microRNA expression profiling studies
}

\author{
XIAOWEI WANG \\ Department of Radiation Oncology, Washington University School of Medicine, St. Louis, Missouri 63108, USA
}

\begin{abstract}
MicroRNAs (miRNAs) are newly identified small noncoding RNA molecules that play important regulatory roles in many biological processes. Changes in miRNA expression are associated with a variety of normal physiological processes as well as with human diseases including cancer. Thus, one important way to characterize miRNA function is to identify changes in miRNA expression profiles. To date, microarrays are the most widely used technique for miRNA expression profiling analysis. However, there are major limitations when microarrays are applied to miRNA profiling studies. Here we report a new PCRbased assay platform for miRNA expression profiling analysis. The primers associated with these miRNA assays were designed using a novel bioinformatics algorithm that has incorporated many primer selection features for improved assay specificity, sensitivity, and homogeneity. The primer design algorithm has been extensively validated experimentally and has been shown to have robust performance for the measurement of miRNA expression in a variety of biological samples at a low cost.
\end{abstract}

Keywords: microRNA; real-time PCR; expression profiling

\section{INTRODUCTION}

MicroRNAs (miRNAs) are noncoding RNAs that regulate many important biological processes (Ambros 2004; Miska 2005). Over 600 human miRNAs have been identified in recent years using both computational and experimental approaches (Griffiths-Jones et al. 2006). A typical mature miRNA molecule is only $\sim 22$-nucleotides (nt) long, which is much shorter than the length of most other gene sequences in humans. Despite the short sequence length and the relatively small number of miRNAs, recent studies have shown that thousands of human protein-coding genes are regulated by miRNAs (Lewis et al. 2005; Lim et al. 2005; Wang and Wang 2006). Thus, deregulated expression of miRNAs may have a profound effect on miRNA-mediated biological pathways.

One major challenge in miRNA research is to accurately and conveniently determine the expression level of miRNAs in various experimental systems. As deregulated expression of miRNAs often indicates functional deregulation, screening for miRNAs with altered expression profiles is likely to

Reprint requests to: Xiaowei Wang, Department of Radiation Oncology, Washington University School of Medicine, St. Louis, MO 63108, USA; e-mail: xwang@radonc.wustl.edu; fax: (314) 362-8521.

Article published online ahead of print. Article and publication date are at http://www.rnajournal.org/cgi/doi/10.1261/rna.1460509. provide clues for the involvements of miRNAs in disease development. In particular, miRNA expression has been extensively studied in human cancers, and altered miRNA expression signatures have been demonstrated to be promising cancer biomarkers (Jay et al. 2007; Yu et al. 2007). At present, this is a field under intense study, and many studies have been published in recent years on using miRNA expression profiles for cancer diagnosis and prognosis. To date, most miRNA expression profiling studies had relied on miRNA microarrays (Yin et al. 2008). Despite their wide use for miRNA profiling, there are some major limitations associated with miRNA microarrays. For example, a large amount of high-quality RNA samples are usually required for the microarray experiments, which is often a major challenge for miRNA expression analysis of clinical samples. In addition, microarray detection specificity is still a major issue because miRNA molecules are very short and many miRNAs differ by only one or a few bases. Another practical consideration is the high cost of microarrays, which discourages the extensive use of microarrays to profile miRNA expression in many biological samples.

Given the relatively small number of miRNAs in humans, it is possible to perform real-time polymerase chain reaction (PCR) in lieu of microarrays for miRNA expression profiling. Several design strategies have been proposed in recent years for PCR-based miRNA detection 
(for review, see Kong et al. 2009). Most of these assays were originally designed to analyze the expression of one or a few miRNAs at a time. However, there are some important primer design considerations when multiple miRNAs are analyzed simultaneously in a single profiling experiment. For example, the primers should have homogenous properties so that all the assays can be performed with similar efficiency under the same experimental condition. More importantly, the primers should be highly specific for the targeted miRNAs with little cross-reactivity to other unintended targets. Unfortunately, most existing primer design programs select primers based on a single template of limited genetic complexity (Chen et al. 2002), leading to frequent nonspecific PCR amplifications. In addition, some important design criteria, such as those for the prevention of primer dimer formation, have not been adequately considered. Thus, an investigator often has to identify primers for genes of interest by trial and error.

To address these potential issues, we have developed a new bioinformatics algorithm for miRNA primer design by incorporating multiple design filters to improve miRNA assay specificity, sensitivity, and homogeneity. This algorithm has incorporated multiple design features that have been proven to be important for PCR performance in our previous studies (Wang and Seed 2003a). In addition, the new design algorithm also includes novel primer features that are uniquely associated with miRNA assays. These new miRNA assays rely on fluorescent detection of amplified PCR products by sequence nonselective dye, SYBR Green I. We have experimentally validated 96 miRNA assays designed with this new algorithm for cancer-related miRNA expression profiling, and the validation results indicate that these new assays have robust performance for the measurement of miRNA expression profiles in a variety of biological samples.

\section{RESULTS}

\section{Overall experimental strategy}

One unique challenge in miRNA assay development is the design of specific reverse transcription (RT) and PCR primers for these very small molecules $(\sim 22 \mathrm{nt})$. A typical mRNA molecule is more than 1-kb long, and it is relatively easy to select gene-specific sequence regions for primer design. In contrast, the design space is very limited for miRNA primers since the miRNA length is the same as that of a typical PCR primer.

The overall experimental strategy using these miRNA assays is summarized in Figure 1. As the first step of the experiment, multiplexed RT reaction is performed using a pool of RT primers. These RT primers anneal specifically to the target miRNAs, leading to a pool of mixed cDNA products from the miRNAs of interest. By performing a multiplexed RT reaction, the overall experimental cost is significantly reduced since only one RT reaction is needed for each RNA sample. After the RT reaction, separate realtime PCR assays are performed to quantify the expression of individual miRNAs. Each PCR is performed with two primers that are uniquely associated with the miRNA of interest, one with a miRNA-specific sequence and the other from the unique tag sequence in the RT primer. Thus, these primers are not likely to cross-react to primers from other miRNA assays even though multiplexed RT reactions are performed. Many bioinformatics parameters have been included in the design algorithm to avoid primer crossreactivity and potential primer dimer and secondary structure formation, as well as to ensure uniform PCR assay properties, such as primer Tm, length, GC content, and the amplicon length. In this way, all miRNA assays can be performed together under the same experimental condition. The overall workflow for the new bioinformatics primer design algorithm is summarized in Figure 2 and described in details below.

\section{Partition of the miRNA sequence}

One critical component of the design algorithm is the proper partition of the mature miRNA sequence. The $3^{\prime}$ end sequence of the miRNA is used for the design of the RT primer, and the $5^{\prime}$-end sequence is used for the design of the PCR reverse primer. Because mature miRNAs are very short, it is usually not possible to identify long nonoverlapping miRNA-specific bases for primer annealing in both RT and PCR. To partly address this issue, the RT reaction is performed at a low temperature $\left(25^{\circ} \mathrm{C}\right)$ so that only a short stretch of miRNA-specific nucleotides are needed for annealing. The number of miRNA-specific bases in an RT primer is mainly determined by two factors, the melting temperature $(\mathrm{Tm})$ requirement and the potential of forming primer dimer. All RT primers contain 7-10 miRNA-specific bases.

It has been well known that primers with the same length may have very different $\mathrm{Tm}$ values. The most accurate way to date to determine the $\mathrm{Tm}$ is the nearest neighbor method (Sugimoto et al. 1995; SantaLucia 1998), which is used in our design program to calculate primer Tm values. The Tm is calculated with the following formula:

$$
\mathrm{Tm}=\Delta \mathrm{H}^{\circ} /\left[\Delta \mathrm{S}^{\circ}-\mathrm{R} \ln \left(\mathrm{C}_{\mathrm{T}} / 4\right)\right],
$$

where $\mathrm{R}$ is the gas constant $(1.987 \mathrm{cal} / \mathrm{K} \mathrm{mol}), \mathrm{C}_{\mathrm{T}}$ is the primer concentration, $\Delta \mathrm{H}^{\circ}$ is the enthalpy change, and $\Delta \mathrm{S}^{\circ}$ is the entropy change. $\Delta \mathrm{H}^{\circ}$ and $\Delta \mathrm{S}^{\circ}$ are calculated with the published thermodynamic parameters (Sugimoto et al. 1995; SantaLucia 1998). The design program initially selects seven miRNA-specific bases for the RT primer. If the calculated Tm for these miRNA-specific bases is lower than $20^{\circ} \mathrm{C}$, then more miRNA-specific bases will be added to the $\mathrm{RT}$ primer until the Tm requirement is met. In this way, all 
Step 1. Multiplexed RT reaction

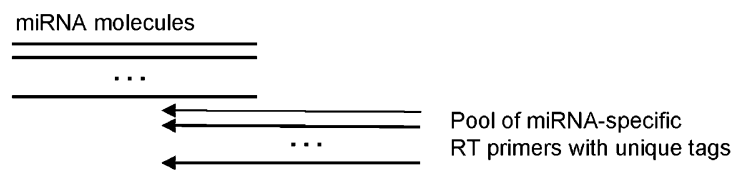

Step 2. Separate real-time $P C R$ reactions
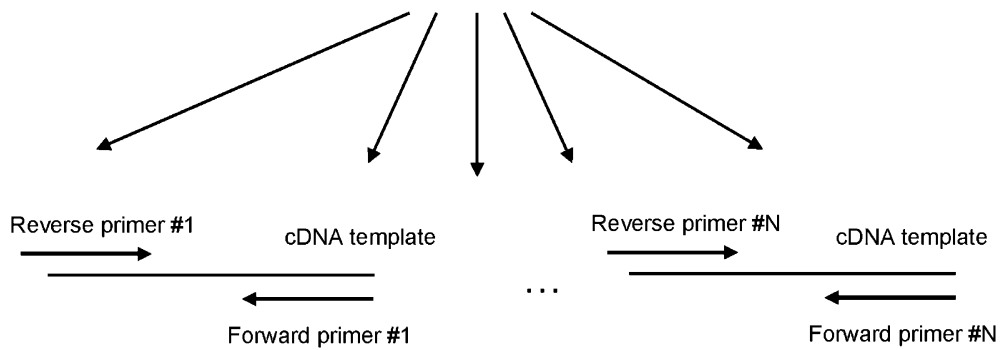

FIGURE 1. The experimental strategy for a new miRNA expression profiling platform with real-time RT-PCR. Multiplexed RT is performed with a pool of miRNA-specific RT primers. These RT primers contain unique tag sequences at the $5^{\prime}$-end. Real-time PCR is then performed with the cDNA templates generated from the multiplexed RT reaction. The PCR reverse primer specifically anneals to the $3^{\prime}$-end of the cDNA template, and the PCR forward primer specifically anneals to the tag sequence in the RT primer.

miRNAs can be efficiently annealed and reverse transcribed under the same RT condition.

Besides the Tm requirement, another important consideration is the avoidance of potential primer dimer formation. This is especially a serious issue for the sequenceindependent fluorescence detection method, such as SYBR Green real-time PCR. After the RT reaction, most RT primers are still left intact in the reaction mixture. Thus, the RT primers could potentially cross-hybridize to PCR primers, leading to a hetero-primer dimer. It is also possible that an RT or PCR primer could self-anneal, leading to a homo-primer dimer. The primer $3^{\prime}$-end residues are especially important for controlling primer dimer formation because DNA polymerase extension can be greatly reduced by mismatches (Kwok et al. 1990; Huang et al. 1992). In our design program, the last four bases of an RT primer should not anneal perfectly to itself or to the PCR primers. If this requirement is not met, then a new partition of the miRNA sequence would be evaluated.

After the successful partition of the miRNA sequence, the $3^{\prime}$-ends of both the RT and PCR reverse primers have been determined. The full RT and reverse primer sequences are then designed by adding extra sequence tags as described in details below.

\section{Design of the PCR reverse primer}

After the partition of the miRNA sequence, only part of the miRNA bases can be used for reverse primer design. In general, this is not enough for specific primer annealing under standard PCR conditions. To address this issue, extra random nucleotides are added to the $5^{\prime}$-end of the reverse primer to increase its $\mathrm{Tm}$. In the end, the primer length is in the range of 1824. Multiple design criteria are used for the addition of these extra nucleotides. The Tm range for all reverse primers is $61^{\circ} \mathrm{C}-63^{\circ} \mathrm{C}$. The $\mathrm{Tm}$ value is calculated using the nearest neighbor method as described above. To avoid potential primer cross-reactivity due to low sequence complexity, a stretch of four or more continuous same nucleotides is not allowed. The newly added extra nucleotides could potentially crosshybridize to miRNA-specific bases in the reverse and RT primers, leading to a primer dimer. Therefore, these added nucleotides are screened against the miRNA sequence for potential dimer formation. The design algorithm also considers primer secondary structure, because a high degree of secondary structure can retard primer annealing, leading to reduced PCR efficiency. In addition, secondary structures may also contribute to nonspecific PCR amplification. Multiple secondary structure filters are used in the algorithm for primer screening as we described previously (Wang and Seed 2003a). A candidate sequence tag is rejected if any one of these design criteria is not met.

\section{Design of the PCR forward primer and the RT primer}

The experimental strategy requires a unique sequence tag in the RT primer sequence. This sequence tag is used as template for PCR forward primer annealing. Thus, the successful design of the forward primer also determines the tag sequence and completes the design of the RT primer. Random 22-mer sequences are generated and evaluated for their qualification as the forward primer using multiple design criteria. In this way, each miRNA assay has a forward primer that is uniquely associated with the assay. This sequence length is long enough to permit generation of assay-specific primers, while reducing the potential for cross-hybridization and allowing cost-effective generation of large primer sets.

As the forward primer does not contain any miRNAspecific bases, more stringent screening criteria can be applied to evaluate a large number of random sequences in the primer selection process. A primer candidate would be discarded if any one of the criteria is not met. The Tm range for the forward primer is $61^{\circ} \mathrm{C}-63^{\circ} \mathrm{C}$, the same as that for the reverse primer as determined by the nearest neighbor method. Similarly, other design filters described earlier for the reverse primer are also applied to the design of the forward primer. To further enhance primer sequence 


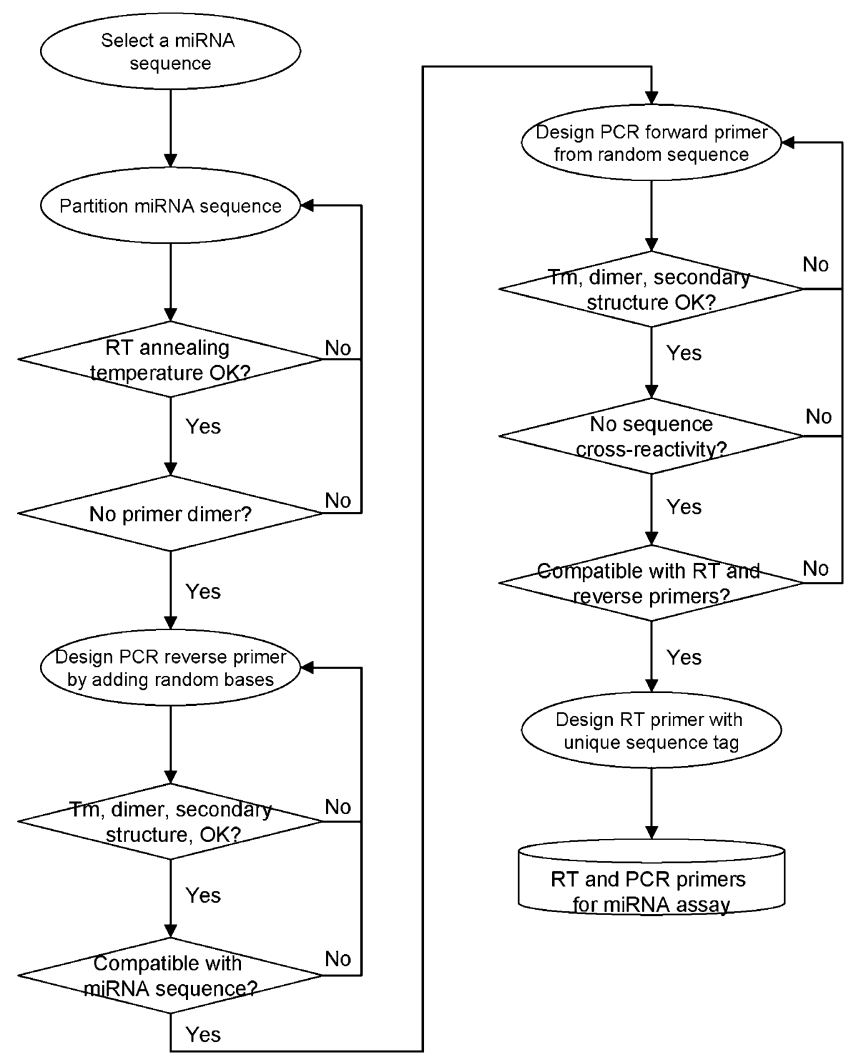

FIGURE 2. A flowchart for the primer design program for the realtime RT-PCR assays.

complexity, the DUST program is used and any sequence identified by DUST is rejected (Hancock and Armstrong 1994). In addition, the GC content is in the range of $45 \%-$ $55 \%$ to ensure uniform priming. The 3 '-end nucleotides contribute most to nonspecific primer extension, especially if the binding of these residues is relatively stable (Rychlik 1995). Thus, the design program evaluates the $\Delta \mathrm{G}$ value for the last five residues at the $3^{\prime}$-end and a threshold value of $-9 \mathrm{kcal} / \mathrm{mol}$ is used for sequence rejection.

Total RNA is typically used in real-time RT-PCR for miRNA quantification. As total RNA contains thousands of distinct transcripts, the likelihood of primer crosshybridization to unintended templates is greatly increased. To address this issue, multiple specificity filters are included in the design algorithm. One main filter is the rejection of sequences with a 15 -mer perfect match to any unintended human genes in the genome. The identification of redundant 15 -mers is performed using a bioinformatics algorithm that we developed previously (Wang and Seed $2003 \mathrm{~b}$ ). To further reduce cross-reactivity, BLAST search is performed against all known human genes, and a threshold BLAST score of 30 is used for primer rejection (threshold value recommended from previous studies) (Wang and Seed 2003b). As the last step of PCR forward primer design, primer candidates are evaluated against the RT primer and the reverse primer. A candidate sequence is rejected if it could potentially cross-react to other primers in the assay. Because of these stringent requirements, thousands of candidate sequences were rejected during the design process before a qualified sequence was found as the forward primer. Finally, the RT primer is designed by attaching the complementary sequence of the forward primer to the miRNA-specific nucleotides as determined by the miRNA sequence partition process.

\section{Experimental evaluation of the miRNA assays}

A small-scale validation experiment was first performed to evaluate the specificity of these miRNA assays using two synthetic miRNAs (miR-124a and let-7a) and five miRNA assays. As shown in Figure 3A, miR-124a was only detected by the miR-124a real-time PCR assay, but not by any other assay included in the study; let-7a was sensitively detected by the let-7a assay. let-7c and let-7b miRNAs are different from let-7a by one and two nucleotides, respectively. Despite the small sequence variations, let-7a RNA was not detected by the assay designed for let- $7 \mathrm{~b}$ and there was only $3 \%$ cross-reactivity to let-7a miRNA using the let-7c assay. Therefore, these miRNA assays have a higher specificity than existing miRNA microarray platforms, which cannot detect a single nucleotide variation.

Next, we identified a set of 96 cancer-related miRNAs by bioinformatics data analysis of public microarray data as well as literature survey. The expression changes of these miRNAs have been shown to be associated with various cancers in previous studies. Real-time RT-PCR assays were designed using the new design algorithm for these cancerrelated miRNAs, and the primer sequences are summarized in Supplemental Table 1. At present, the normalization of miRNA expression profiling data is challenging, and there is no consensus on the best normalization strategy. Commonly used methods for miRNA raw data processing include median or mean normalization, which uses median or mean value of the raw readings as the normalization factor to correct for intersample signal variation. However, many miRNAs may not be expressed in a biological sample, and thus median or mean value may be skewed toward the assay readings for lowly expressed miRNAs, which tend to be more variable compared with the readings for more abundantly expressed miRNAs. In our analysis, a new normalization strategy was adopted. Instead of using the plate median or mean for normalization, the readings from eight miRNAs with relatively high expression were averaged and the mean value was used as the plate normalization factor (see Materials and Methods for details). This normalization strategy was used in all subsequent analyses of the expression profiles of 96 cancer-specific miRNAs.

The assay specificity was further evaluated with two cell lines using 96 cancer-related miRNA assays. One cell line is ovarian cancer SKOV-3, and the other is SKOV-3-miR-146a, 
A

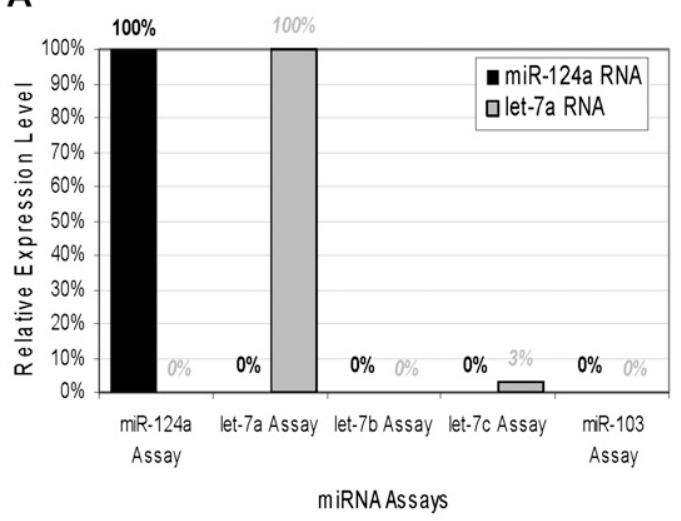

B

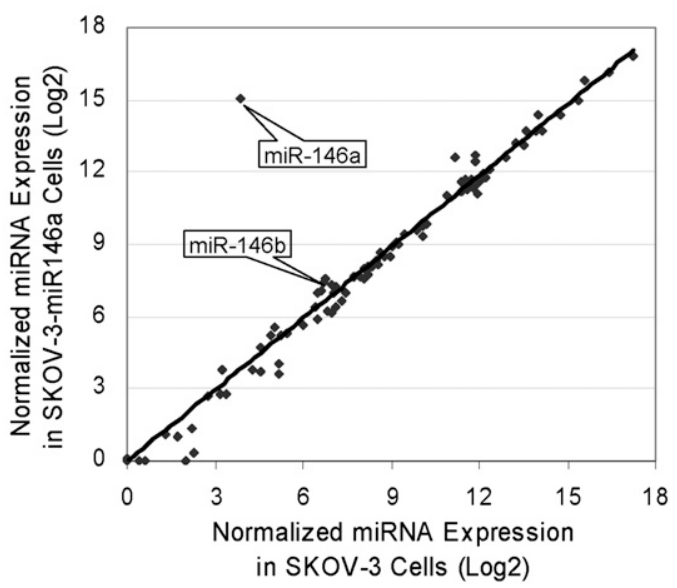

FIGURE 3. Real-time RT-PCR validation of the miRNA assays. $(A)$ The assay specificity was evaluated with two synthetic miRNAs, miR124a and let-7a. Five miRNA assays were evaluated for the detection of these synthetic miRNAs. The assay readings of miR-124a by miRNA-124a assay, and let-7a by let-7a assay were used as the relative standards (100\%) for the analysis of assay cross-reactivity. (B) The assay specificity was evaluated with two cell lines and 96 cancerrelated miRNA assays. One cell line is SKOV-3 and the other one is SKOV-3-miR-146a, which is SKOV-3 with stably expressed miR-146a. Each data point represents the normalized readings from one miRNA assay in both cell lines.

which is SKOV-3 with a stably expressed miR-146a using a lentiviral expression system. As shown in Figure 3B, miR146a was overexpressed in SKOV-3-miR146a cells by over 2000 times compared with SKOV-3 cells. Interestingly, the detection of other miRNAs was little affected by miR-146a overexpression. The miRNA expression profiles between the two cell lines were highly correlated after excluding miR-146a from the calculation (Pearson $r=0.99$ ). Most noticeably, although miR-146b differs from miR-146a by a single nucleotide, its expression detection was not affected by the high abundance of miR-146a (Fig. 3B, crossreactivity $<0.2 \%$ ). In a similar way, we also compared SKOV-3 cells with SKOV-3-miR-200a cells (SKOV-3 with overexpressed miR-200a). As in the case of miR-146a, overexpression of miR-200a did not affect the quantification of other miRNAs, including two highly homologous miRNAs from the same miR-200 family, miR-200c and miR-141 (data not shown).

The assay specificity was also evaluated with frozen tumor tissue samples. As shown in Figure 4B, there was little nonspecific amplification from negative control RT reactions (using Escherichia coli total RNA as the RT template) from all 96 miRNA assays. In contrast, most miRNAs of interest were specifically amplified in cervical tumor tissues with high PCR efficiency (Fig. 4A). Melting curve analysis (plotted as the first derivatives of absorbance in relation to temperature) was also performed to evaluate the specificity of the PCR products. Multiple peaks in a melting curve usually represent mixed PCR products including nonspecific amplicons. As shown in Figure 4C, all the melting curves for the amplified products contain single peaks, indicating specific PCR amplification. The PCR product size was further checked by agarose gel electrophoresis, and the expected amplicon sizes were observed from all the PCR assays (data not shown).

Having successfully validated the miRNA assays with cell lines and frozen tumor tissues, we then evaluated whether these assays can be robustly applied to profile miRNA expression in formalin-fixed paraffin-embedded (FFPE) tissues. RNA extracted from FFPE tissues is usually highly degraded and challenging to apply to microarrays. We first determined the miRNA expression profiles from two consecutive sections from the same tumor block. Similar to the result shown in the cell line comparison analysis, the miRNA expression profiles of the two tissue sections were highly correlated $(r=0.99)$. Thus, the profiling result was highly reproducible for FFPE tissue analysis. To further explore the usefulness of these new miRNA assays, we profiled four FFPE breast tumors, including two high-grade and two low-grade tumors. The two miRNA expression profiles from each group (high grade or low grade) were averaged, and differentially expressed miRNAs between the two groups are presented in Figure 5. Several miRNAs were overexpressed in high-grade tumors, and some of these miRNAs are already known to promote tumorigenesis. For example, the miR-17-92 cluster has been shown by many studies to promote tumor growth (He et al. 2005). Interestingly, three miRNAs from this cluster were found to be overexpressed in high-grade breast tumors in our analysis (miR-17, miR-18, and miR-20). Another interesting overexpressed miRNA is miR-21, which is already known to be overexpressed in almost all human cancers. The most overexpressed miRNA in our analysis, miR-210 has recently been shown to be an independent prognostic marker in breast cancer (Camps et al. 2008). On the other hand, miRNAs underexpressed in high-grade tumors included several known tumor suppressors. For example, let-7 miRNAs are known to suppress the progression of 
A

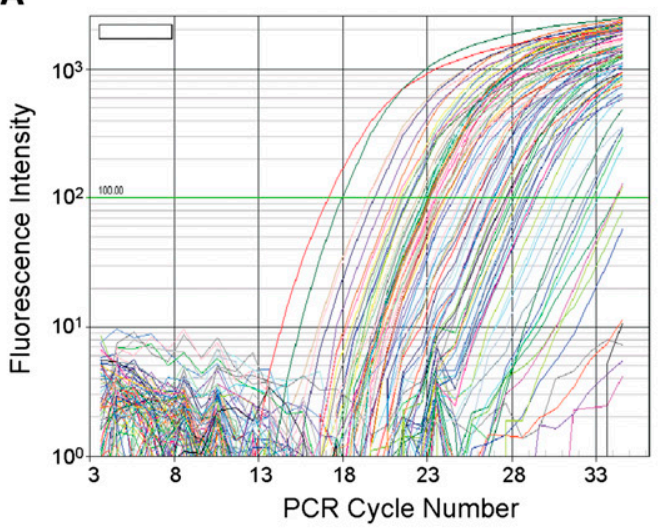

B

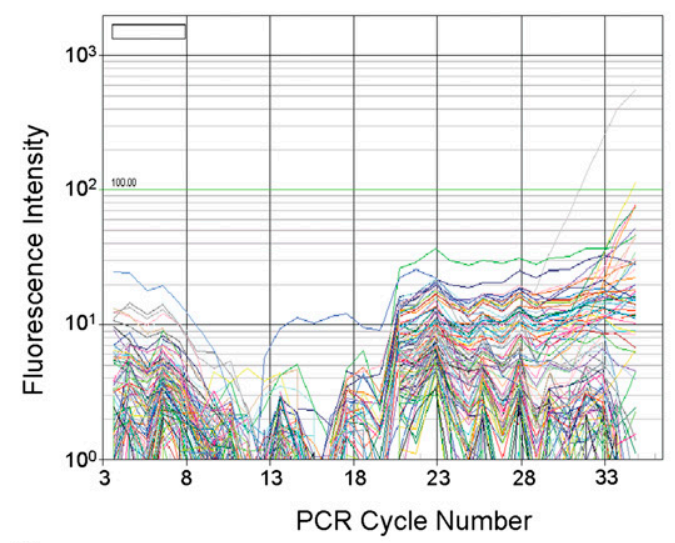

C

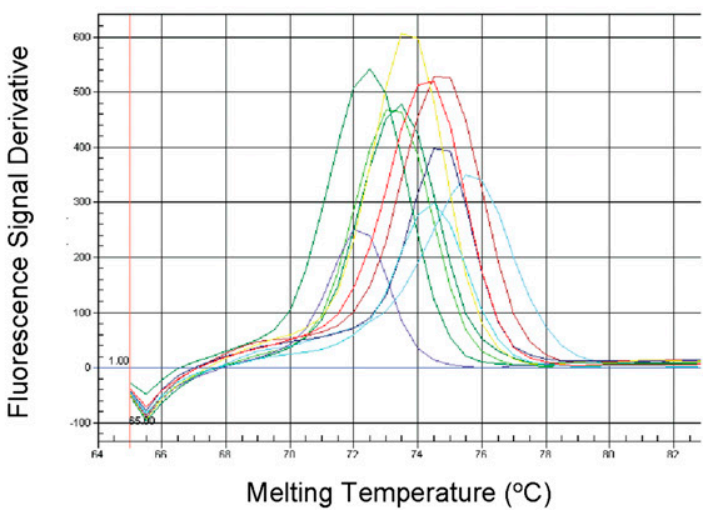

FIGURE 4. Real-time RT-PCR validation of 96 cancer-related miRNA assays. (A) Real-time RT-PCR was performed with the total RNA extracted from a frozen cervical tumor tissue. Each line in the plot represents the amplification curve for one miRNA. (B) Real-time RT-PCR was performed with the total RNA from E. coli. (C) Melting curve analysis of the real-time PCR profile for the cervical tissue miRNAs. For illustration purpose, only 10 dissociation curves are displayed here for 10 of the 96 miRNA assays. Other dissociation curves (data not shown) were similar to those presented here.

many human cancers (Roush and Slack 2008); tumor suppressor miR-34 miRNAs are required for p53-mediated cell death (He et al. 2007). Thus, our preliminary analysis of four FFPE tumor tissues suggests that the new miRNA assays could be used as a reliable tool to systematically study miRNA expression in FFPE samples.

\section{DISCUSSION}

Microarrays are by far the most widely used technique for transcriptional expression profiling studies. Microarrays are routinely used to simultaneously measure the expression of tens of thousands of mRNA transcripts in a single experiment, and thus the experimental cost per measurement is greatly reduced. Microarrays have also been successfully applied to miRNA expression profiling analysis, and multiple microarray platforms have been proposed in recent years for miRNA studies. To date, most published miRNA profiling studies were performed with microarrays.

Despite their popularity, there are also major issues associated with miRNA microarrays. One concern is the throughput of miRNA microarrays. There are only several hundred known miRNAs in humans. Therefore, there is little cost-saving benefit when microarrays are applied to miRNA studies. On the other hand, microarrays are a solution hybridization-based detection technology, and the signals are generally considered to be more noisy and less specific than that from real-time PCR. Thus in general real-time PCR experiments have to be performed to subsequently validate the findings from microarrays. Given the relatively low-throughput nature of miRNA profiling analysis, real-time RT-PCR would in theory be ideal when both experimental cost and assay reliability are considered. Unfortunately, real-time RT-PCR has not been widely adopted to date for miRNA profiling analysis. One major roadblock is the availability of cheap and reliable miRNA assays. Our study is an attempt to overcome this obstacle. Currently, one popular PCR-based method for miRNA quantification is based on TaqMan chemistry (Chen et al. 2005). In a TaqMan qPCR assay, two PCR primers and one TaqMan probe are required. In contrast, our PCR assays are based on sequence-independent fluorescence detection using SYBR Green. This is to significantly reduce the experimental cost because SYBR Green real-time PCR is by far the cheapest and most widely used PCR quantification method. A miRNA assay design strategy has recently been proposed based on SYBR Green real-time PCR (Sharbati-Tehrani et al. 2008). The qPCR assay requires one miRNA-specific primer and two universal primers for the analysis of one or a few miRNAs in one experiment (Sharbati-Tehrani et al. 2008). In contrast, our assay primers are designed using a robust bioinformatics algorithm so that multiplexed RT-PCR can be performed for miRNA expression profiling. Many design features have been implemented in both the experimental assay strategy and the primer design algorithm for assay specificity, sensitivity, and homogeneity. The assay design has been extensively validated experimentally for 96 cancerrelated miRNA assays, and the results indicate that 


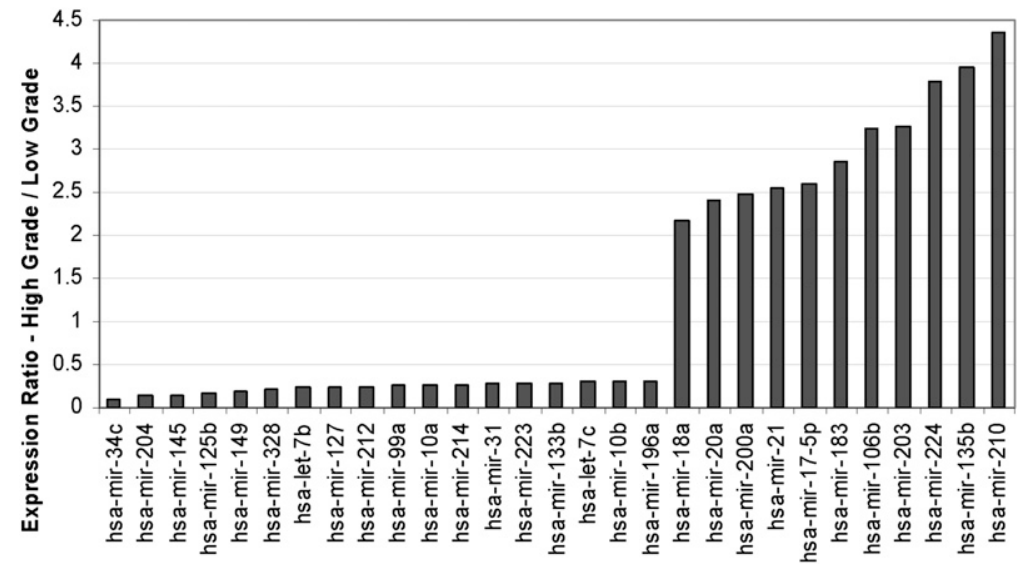

FIGURE 5. miRNA expression profiling of FFPE breast tumor tissues. The expression ratio is defined as the miRNA expression fold change between the high-grade and low-grade tumors. miRNAs with the most extreme expression ratios $(>2$ or $<0.5)$ are presented here. included $1 \mu \mathrm{L}$ of $10 \times \mathrm{RT}$ buffer, $0.4 \mu \mathrm{L}$ of $25 \times$ dNTP $(100 \mathrm{mM}), 0.4 \mu \mathrm{L}$ of RT primer mixture $(250 \mathrm{nM}), 0.5 \mu \mathrm{L}$ of reverse transcriptase, $0.5 \mu \mathrm{L}$ of RNase inhibitor, $2.2 \mu \mathrm{L}$ of $\mathrm{H}_{2} \mathrm{O}$, and $5 \mu \mathrm{L}$ of RNA template (20-100 ng). The RT reaction mixture was incubated for $20 \mathrm{~min}$ at $25^{\circ} \mathrm{C}$ and for $60 \mathrm{~min}$ at $37^{\circ} \mathrm{C}$ and was finally heat-inactivated for $5 \mathrm{~min}$ at $85^{\circ} \mathrm{C}$. Real-time PCR was performed with power SYBR Green PCR master mix (Applied Biosystems). The real-time PCR running protocol was $10 \mathrm{~min}$ at $95^{\circ} \mathrm{C}$, followed by three cycles of amplification $\left(15 \mathrm{sec}\right.$ at $95^{\circ} \mathrm{C}$, $1 \mathrm{~min}$ at $55^{\circ} \mathrm{C}$, and $30 \mathrm{sec}$ at $60^{\circ} \mathrm{C}$ ). Then 32 additional amplification cycles were performed $\left(10 \mathrm{sec}\right.$ at $95^{\circ} \mathrm{C}$ and $30 \mathrm{sec}$ at $\left.60^{\circ} \mathrm{C}\right)$. In this way, miRNA expression in each tumor sample was profiled using a single 96-well PCR plate.

In order to directly compare the expresthese new miRNA assays can be reliably applied to a variety of RNA samples including challenging clinical samples with minute amounts of highly degraded RNA. With these new assays, the experimental cost for the profiling of 96 cancer-related miRNAs is only less than one-tenth compared with that of microarrays. Also importantly, the high measurement accuracy of these miRNA assays will save researchers valuable time and resources down the road for the validation of the profiling results. To promote the idea of using real-time RT-PCR for miRNA expression profiling, we have made freely available all the primer sequences of our validated miRNA assays. It is likely that in the near future more and more miRNA profiling analyses will be performed using real-time RT-PCR instead of microarrays.

\section{MATERIALS AND METHODS}

\section{RNA samples}

A variety of RNA samples were analyzed to evaluate the performance of the new miRNA assays. Synthesized miRNA oligonucleotides were purchased from Integrated DNA Technologies (IDT). For the cell line comparison analysis, SKOV-3 cancer cells were stably transfected with miR-146a or miR-200a constructs using a lentiviral expression system according to the manufacturer's protocol (System Biosciences). Total RNA samples from cells or frozen tissues were prepared with a mirVana miRNA isolation kit (Ambion). FFPE tissue section slides were used to extract total RNA from four breast tumors (two grade I and two grade III tumors) using the miRNeasy FFPE Kit for miRNA isolation (Qiagen).

\section{Real-time RT-PCR}

All the DNA oligonucleotide primers were synthesized by IDT. RT reactions were performed with a high-capacity cDNA reverse transcription kit (Applied Biosystems). Each $10 \mu \mathrm{L}$ RT reaction sion profiles of different RNA samples, raw expression values from the real-time PCR experiments were normalized using a new analysis strategy. First, the expression values of 96 miRNAs from an RNA sample were sorted so that the first miRNA had the highest expression reading and the last miRNA had the lowest reading. Next, a plate normalization factor $n$ was calculated by averaging eight selected miRNA expression values as the following:

$$
n=\frac{1}{8} \sum_{i=2}^{9} E_{5 i}
$$

where $E$ represents the miRNA expression reading and the subscript $5 i$ represents the order of eight selected miRNAs in the sorted list (i.e., 10th, 15th ...45th miRNAs on the list). Finally, normalized expression values in each PCR plate were calculated by dividing raw expression values with the plate normalization factor $n$.

\section{SUPPLEMENTAL MATERIAL}

Supplemental material can be found at http://www.rnajournal.org.

\section{ACKNOWLEDGMENTS}

I thank Xiaoxia Hu for technical assistance. I also thank Qin Yang, Perry Grigsby, and Yin-yuan Mo for providing the tumor tissues and lentiviral constructs. This research was supported by a startup fund from the Washington University School of Medicine in St. Louis.

Received November 11, 2008; accepted December 19, 2008.

\section{REFERENCES}

Ambros, V. 2004. The functions of animal microRNAs. Nature 431: 350-355.

Camps, C., Buffa, F.M., Colella, S., Moore, J., Sotiriou, C., Sheldon, H., Harris, A.L., Gleadle, J.M., and Ragoussis, J. 2008. 
hsa-miR-210 Is induced by hypoxia and is an independent prognostic factor in breast cancer. Clin. Cancer Res. 14: 1340-1348. Chen, B.Y., Janes, H.W., and Chen, S. 2002. Computer programs for PCR primer design and analysis. Methods Mol. Biol. 192: 19-29.

Chen, C., Ridzon, D.A., Broomer, A.J., Zhou, Z., Lee, D.H., Nguyen, J.T., Barbisin, M., Xu, N.L., Mahuvakar, V.R., Andersen, M.R., et al. 2005. Real-time quantification of microRNAs by stem-loop RT-PCR. Nucleic Acids Res. 33: e179.

Griffiths-Jones, S., Grocock, R.J., van Dongen, S., Bateman, A., and Enright, A.J. 2006. miRBase: MicroRNA sequences, targets, and gene nomenclature. Nucleic Acids Res. 34: D140-D144.

Hancock, J.M. and Armstrong, J.S. 1994. SIMPLE34: An improved and enhanced implementation for VAX and Sun computers of the SIMPLE algorithm for analysis of clustered repetitive motifs in nucleotide sequences. Comput. Appl. Biosci. 10: 67-70.

He, L., Thomson, J.M., Hemann, M.T., Hernando-Monge, E., Mu, D., Goodson, S., Powers, S., Cordon-Cardo, C., Lowe, S.W., Hannon, G.J., et al. 2005. A microRNA polycistron as a potential human oncogene. Nature 435: 828-833.

He, L., He, X., Lowe, S.W., and Hannon, G.J. 2007. microRNAs join the 553 network: Another piece in the tumour-suppression puzzle. Nat. Rev. Cancer 7: 819-822.

Huang, M.M., Arnheim, N., and Goodman, M.F. 1992. Extension of base mispairs by Taq DNA polymerase: Implications for single nucleotide discrimination in PCR. Nucleic Acids Res. 20: 45674573.

Jay, C., Nemunaitis, J., Chen, P., Fulgham, P., and Tong, A.W. 2007. miRNA profiling for diagnosis and prognosis of human cancer. DNA Cell Biol. 26: 293-300.

Kong, W., Zhao, J.J., He, L., and Cheng, J.Q. 2009. Strategies for profiling microRNA expression. J. Cell. Physiol. 218: 22-25.

Kwok, S., Kellogg, D.E., McKinney, N., Spasic, D., Goda, L., Levenson, C., and Sninsky, J.J. 1990. Effects of primer-template mismatches on the polymerase chain reaction: Human immunodeficiency virus type 1 model studies. Nucleic Acids Res. 18: 9991005.
Lewis, B.P., Burge, C.B., and Bartel, D.P. 2005. Conserved seed pairing, often flanked by adenosines, indicates that thousands of human genes are microRNA targets. Cell 120: 15-20.

Lim, L.P., Lau, N.C., Garrett-Engele, P., Grimson, A., Schelter, J.M., Castle, J., Bartel, D.P., Linsley, P.S., and Johnson, J.M. 2005. Microarray analysis shows that some microRNAs down-regulate large numbers of target mRNAs. Nature 433: 769-773.

Miska, E.A. 2005. How microRNAs control cell division, differentiation and death. Curr. Opin. Genet. Dev. 15: 563-568.

Roush, S. and Slack, F.J. 2008. The let-7 family of microRNAs. Trends Cell Biol. 18: 505-516.

Rychlik, W. 1995. Priming efficiency in PCR. Biotechniques 18: 84-86, 88-90.

SantaLucia Jr., J. 1998. A unified view of polymer, dumbbell, and oligonucleotide DNA nearest-neighbor thermodynamics. Proc. Natl. Acad. Sci. 95: 1460-1465.

Sharbati-Tehrani, S., Kutz-Lohroff, B., Bergbauer, R., Scholven, J., and Einspanier, R. 2008. miR-Q: A novel quantitative RT-PCR approach for the expression profiling of small RNA molecules such as miRNAs in a complex sample. BMC Mol. Biol. 9: 34.

Sugimoto, N., Nakano, S., Katoh, M., Matsumura, A., Nakamuta, H., Ohmichi, T., Yoneyama, M., and Sasaki, M. 1995. Thermodynamic parameters to predict stability of RNA/DNA hybrid duplexes. Biochemistry 34: 11211-11216.

Wang, X. and Seed, B. 2003a. A PCR primer bank for quantitative gene expression analysis. Nucleic Acids Res. 31: e154.

Wang, X. and Seed, B. 2003b. Selection of oligonucleotide probes for protein coding sequences. Bioinformatics 19: 796-802.

Wang, X. and Wang, X. 2006. Systematic identification of microRNA functions by combining target prediction and expression profiling. Nucleic Acids Res. 34: 1646-1652.

Yin, J.Q., Zhao, R.C., and Morris, K.V. 2008. Profiling microRNA expression with microarrays. Trends Biotechnol. 26: 70-76.

Yu, S.L., Chen, H.Y., Yang, P.C., and Chen, J.J. 2007. Unique microRNA signature and clinical outcome of cancers. DNA Cell Biol. 26: 283-292. 

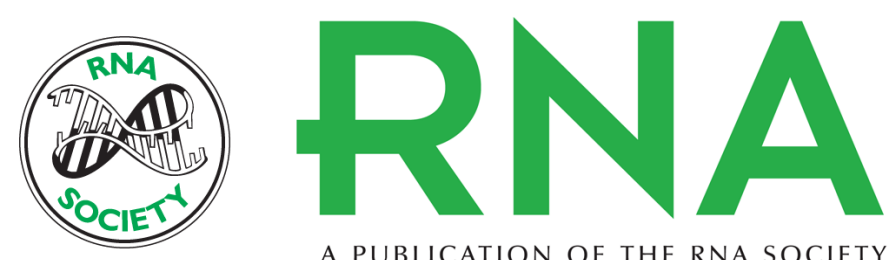

A PUBLICATION OF THE RNA SOCIETY

\section{A PCR-based platform for microRNA expression profiling studies}

\section{Xiaowei Wang}

RNA 2009 15: 716-723 originally published online February 13, 2009

Access the most recent version at doi:10.1261/rna.1460509

Supplemental http://rnajournal.cshlp.org/content/suppl/2009/02/17/rna.1460509.DC1
Material

References This article cites 25 articles, 2 of which can be accessed free at: http://rnajournal.cshlp.org/content/15/4/716.full.html\#ref-list-1

License

Email Alerting Receive free email alerts when new articles cite this article - sign up in the box at the Service top right corner of the article or click here. 